\title{
Distribution Network Design: Selection and Sizing of Congested Connections
}

\author{
Simin Huang, Rajan Batta and Rakesh Nagi \\ Department of Industrial Engineering, 342 Bell Hall, \\ University at Buffalo (SUNY), Buffalo, NY 14260, USA \\ Revised: November 2004
}

April 2004

\begin{abstract}
This paper focuses on certain types of distribution networks in which commodity flows must go through connections that are subject to congestion. Connections serve as transshipment and/or switching points and are modeled as $M / G / 1$ queues. The goal is to select connections, assign flows to the connections, and size their capacities, simultaneously. The capacities are controlled by both the mean and the variability of service time at each connection. We formulate this problem as a mixed integer nonlinear optimization problem for both the fixed and variable service rate cases. For the fixed service rate case, we prove that the objective function is convex and then develop an outer approximation algorithm. For the variable service rate case, both mean and second moment of service time are decision variables. We establish that the utilization rates at the homogeneous connections are identical for an optimal solution. Based on this key finding, we develop a Lagrangian relaxation algorithm. Numerical experiments are conducted to verify the quality of the solution techniques proposed. The essential contribution of this work is the explicit modeling of connection capacity (through the mean and the variability of service time) using a queueing framework.
\end{abstract}

Keywords: Distribution network; Connections; Congestion. 


\section{Introduction}

Certain types of distribution networks require that flows between origin-destination node pairs pass through connections. These connections serve as transshipment and/or switching points. The application we had in mind for a connection is a distribution center (DC) goods flow into the DC from manufacturing plants and are later shipped to fulfill customer demand. Several multinational companies use distribution center networks in this manner. For example, a paper products company needs to distribute paper towels, toilet tissue, napkins, etc. These products are light but are bulky. They cannot be compressed for transportation or storage purposes as they will lose their shape. Transportation and storage cost for such items is very high in comparison to the cost of the items themselves, and their large storage volume causes congestion in the DC. In such an industry setting, selection and sizing of distribution centers is a problem of much significance. We further note that our focus is not on industries where there is relatively less congestion and large economies of scale are realized by traveling through connections; for example, the hub-and-spoke network in an airline industry.

For most fast moving consumer goods, demand patterns are quite erratic, being a function of both sales promotions (i.e., seasonal) and regular demand. In response to this, at the strategic or aggregate level, the production schedules for individual SKUs (Stock Keeping Units) in a shared facility can be characterized as random variates. We can therefore model the flows to a DC as a Poisson process. Items are stored at a DC for an arbitrary period of time and are later retrieved when a customer demand needs to be fulfilled. The residence time in a DC is a function of the extent of supply chain coordination and the degree of 
automation in information and material flow. Much of this is in the control of management, albeit at a cost. We assume a general distribution for the residence time variable. Effectively, we model a $\mathrm{DC}$ as an $M / G / 1$ queueing system and control its capacity through both the mean and the variability of service time at each connection. By a judicious choice of flows of items to DCs we can control these storage delays and also influence transportation cost. We therefore consider the problem of selecting the connections to open, assigning flows to them, and sizing their capacities simultaneously, in order to minimize overall system cost, i.e., the sum of transportation cost, service cost (service time plus waiting time), and connection installation cost (could be a function of its capacity).

We provide a simple numerical example to demonstrate the importance of modeling congestion for this problem. Suppose there are four flows with amount 5 units each and two connection candidates with a fixed charge cost of 10 . The flow travel times by way of connections are as follows:

\section{Flow1 Flow2 Flow3 Flow4}

Connection $1\left(\begin{array}{cccc}1 & 1 & 1 & 1 \\ 2 & 2 & 2 & 2\end{array}\right)$

We compare three alternatives in the example: (i) using a fixed charge facility location model without modeling of congestion; (ii) modeling congestion with a fixed rate; and (iii) modeling congestion with variable rate. Total cost is the sum of transportation cost, service cost (service time plus waiting time), and connection installation cost (see details later). In case (i) and (ii), each connection is an $M / G / 1$ queueing system with fixed mean service time and its second moment. Suppose that the mean service time is 0.048 and its second moment is 0.02. According to the models we present later, we have optimal solutions for these cases 
as follows:

- No modeling of congestion: all flows will go to connection 1 with utilization rate 0.96 and total cost 201.79 .

- Fixed rate: 13 units of flow will go to connection 1 with utilization rate 0.62 and 7 units go to connection 2 with utilization rate 0.34 . The total cost is 194.86 .

- Variable rate: all flows will go to connection 1 with utilization rate 0.60 and total cost 108.65. Its mean service time is 0.03 and the second moment 0.045 .

This simple example illustrates that modeling congestion with variable capacity size (or rate) in such distribution networks can lead to significant saving for the system.

Most earlier work in joint sizing and location are in a deterministic setting (e.g. Huang, Batta, and Nagi (2003)). The originality of the present paper is the explicit modeling of connection capacity (controlled by the mean and the variability of service time) using a queueing framework. The consideration of service time variability is an important issue in the design of a queueing facility. The influence of the variability is illustrated by Hillier and So (1991), Melnyk, Denzler, and Fredendall (1992), and Enns (1998). However, this explicit consideration of capacity introduces numerous technical challenge, since the objective function and the constraints become highly non-linear. The traditional approaches to deal with the difficulties are to simplify models by either assuming constant variance or constant coefficient of variation. The methodology we adopt to address these technical difficulties is unique. We first establish an equal utilization property for the connections and then use this property to drive the solution methodology. Without this approach the problem remains highly intractable. 
Two cases of the problem are considered in this paper. For the fixed service rate case, we assume that the capacity of each connection is unchangeable. In the variable service rate case we assume that the capacity of connections is to be determined. The resulting mathematical formulations are mixed integer nonlinear programs (MINLP). For the fixed service rate case, we establish a convexity property and develop an outer approximation algorithm. For the variable service rate case, we show that the optimal utilization rate at each selected connection should be equal when the cost of unit capacity is independent of connection location. This key finding enables us to find bounds for total number of connections and total service rate. Also, an efficient Lagrangian relaxation algorithm is developed based on these results.

The rest of this paper is organized as follows: Section 2 reviews related research on this problem. Section 3 provides some preliminaries. Section 4 analyzes the fixed service rate case, proves the convexity property of this problem, and develops an outer approximation algorithm for this case. Section 5 details the variable service rate case. We establish that the utilization rates at the connections are identical for an optimal solution along with other properties about how to determine this. Based on this key property, we develop a Lagrangian relaxation algorithm. Section 6 presents computational performance for both algorithms. Finally, Section 7 provides a summary and suggests directions for future work.

\section{Review of Related Literature}

These are several other loosely related models that have been developed in the OR/MS literature that help view our model in a broader context. 
The hub-and-spoke network model presented in O'Kelly (1986) involves locating a set of fully interconnected facilities called hubs, which serve as transshipment points for flows between specified origin and destination nodes. The major assumption in this model is that the travel cost for inter-hub movement is less than the cost for movement between a hub and a spoke (due to economies of scale). Campbell (1994) presents an integer programming formulation for the capacitated hub location problem, in which he takes the hub installation cost into consideration and restricts the sum of the flows entering any hub to be less than the capacity of that hub. Other capacitated hub location problems can be found in Aykin (1994) and Ebery et al. (2000). The difference between the hub-and-spoke network model and ours is that while we do not consider economies of scale for travel cost we do consider the queueing behavior of connections.

Another related work is the flow-capturing (or discretionary service facility) model introduced independently by Berman, Larson, and Fouska (1992) and Hodgon (1990). It is motivated by applications such as locating gas stations. The problem is formulated on a transportation network with customers traveling on pre-planned paths. It assumes that a customer will use a facility only if it lies on the pre-planned path. The objective is to maximize the utilization by customers. The difference between this problem and ours is that the flow-capturing model tries to attract more customers while our models try to spread flows in order to reduce congestion.

There are some other location models that take congestion into consideration. The stochastic queue median (SQM) model due to Berman and Larson (1982) considers locating a mobile server to minimize the average response time. Berman, Larson, and Parkan (1987) develop two heuristics for locating $p$ facilities on a congested network. Batta, Ghose, and 
Palekar (1989) solve this problem for the Manhattan metric with arbitrary shaped barriers and convex forbidden regions. In the SQM problem, the arrival rate at each facility is known and the service rate is a function of facility location. In our case, the arrival rate at each connection is not fixed and the service rates are possible decision variables. Recent facility location problems with congestion can be found in Marianov (2003), who formulates a model to locate multiple-server congestible facilities in order to maximize total expected demand; and Wang, Batta, and Rump (2002), who study a facility location problem with stochastic customer demand and immobile servers to minimize customers' total traveling cost and waiting cost. Again, the sizing of facilities are not considered in these models.

Capacity planning is considered in the design of flexible manufacturing systems. Queueing networks are applied to control the service rate, the number of servers, input rates, etc. There are many pieces of work that use queueing network models e.g., see papers by Yamazaki, Sakasegawa, and Shanthikumar (1992), Yao and Kim (1987), Shanthikumar and Yao (1988), Bitran and Tirupati (1989) and Bretthauer (1995). For a comprehensive review of queueing system design problem the reader is referred to Crabill, Gross, and Magazine (1977) and Stidham (2002). These models only consider what happens at the facilities and are suitable for the design problems where the travel distances between facilities can be ignored.

Brimberg, Mehrez, and Wesolowsky (1997) and Brimberg and Mehrez (1997) consider the allocation of a set of servers across queueing facilities so as to best service demand. This is another way to define the capacity or size of a facility and is hence related to our work. However, we note that our model also considers location of facilities in addition to sizing and flow assignment. 


\section{Preliminaries}

We assume that connections will be selected from a set of candidate sites $K$ and let $|K|=n_{K}$, where $|\cdot|$ denotes a set's cardinality. Let $N$ be set of origins and destinations; their locations are assumed to be given, with $|N|=n_{N}$. Each pair of origin and destination is connected through a connection facility. $A$ is the set of origin-destination pairs. The term $f_{i j}$ represents the flow amount from origin node $i$ to destination node $j$, for $(i, j) \in A$. We assume that $f_{i j}>0$ for all $(i, j) \in A$. This will simplify our presentation of the formulation, proofs and analysis. If some $f_{i j}$ are equal to zero we can reformulate the problem by only defining decision variables for $i, j$ combinations that have $f_{i j}>0$. The terms $d_{i k}$ and $d_{k j}$ represent the shortest distances from node $i$ to connection $k$ and from connection $k$ to node $j$, respectively; $v$ is the travel speed of the flows; $F_{k}$ is the fixed installation cost that is related to connection $k$. We let $\alpha$ be the net present value (see, e.g., Park (2002)) of per unit time per unit flow evaluated over the system's lifetime.

Let $\lambda_{k}, \bar{S}_{k}$, and $\bar{S}_{k}^{2}$ be the total flow, mean service time, and the second moment of service time at connection $k$, respectively. Let $x_{i j k}$ be the fraction of flow $i$ - $j$ by way of connection $k$; and $y_{k}=1$ if a connection is located at candidate site $k$, and 0 otherwise.

We use the notation $Z^{P}(\boldsymbol{x})$ to denote the objective function of a certain problem, where $(P)$ indicates the problem and bold $\boldsymbol{x}$ indicates the vector of decision variables.

\section{The Fixed Service Rate Case}

In this section, we assume that the mean and second moment of service time at each connection are known constants. The goal of the problem here is to select some number of queueing 
connections and assign flows to them.

\subsection{Formulation}

The total arrival rate at connection $k$ is given by: $\lambda_{k}=\sum_{(i, j) \in A} f_{i j} x_{i j k}, \forall k \in K$. Since the system is assumed to operate as an $M / G / 1$ queue, from Gross and Harris (1998), the average waiting time in queue at connection $k$ can be written as: $\frac{\lambda_{k} \bar{S}_{k}^{2}}{2\left(1-\lambda_{k} S_{k}\right)}$. Therefore, the selection of stochastic connections with fixed service rate problem, $\left(P_{1}\right)$, can be formulated as follows:

$$
\begin{gathered}
\left(P_{1}\right) \min _{\boldsymbol{x}, \boldsymbol{y}} Z^{P_{1}}(\boldsymbol{x}, \boldsymbol{y})=\sum_{(i, j) \in A} \sum_{k \in K} \alpha f_{i j}\left(\frac{d_{i k}+d_{k j}}{v}+\frac{\bar{S}_{k}^{2} \sum_{(i, j) \in A} f_{i j} x_{i j k}}{2\left(1-\bar{S}_{k} \sum_{(i, j) \in A} f_{i j} x_{i j k}\right)}+\bar{S}_{k}\right) x_{i j k}+\sum_{k \in K} F_{k} y_{k} \\
\text { subject to } \quad \sum_{k \in K} x_{i j k}=1, \quad \forall(i, j) \in A \\
\quad x_{i j k} \leq y_{k}, \quad \forall(i, j) \in A, \forall k \in K \\
1-\bar{S}_{k} \sum_{(i, j) \in A} f_{i j} x_{i j k} \geq \epsilon, \quad \forall k \in K \\
x_{i j k} \geq 0, y_{k} \in\{0,1\}, \quad \forall(i, j) \in A, k \in K
\end{gathered}
$$

Here $\epsilon$ is a small positive number. Writing constraints (4) as $1-\bar{S}_{k} \sum_{(i, j) \in A} f_{i j} x_{i j k} \geq \epsilon$ instead of $1-\bar{S}_{k} \sum_{(i, j) \in A} f_{i j} x_{i j k}>0$ ensures that $\boldsymbol{X}=\{\boldsymbol{x}: \boldsymbol{x}$ satisfies constraints (4) and $\boldsymbol{x} \geq 0\}$ is a compact set. The objective function (1) minimizes the total travel cost, average waiting time and service time cost at the connections, and the fixed installation cost of the connections. Constraints (2) stipulate that the flows only travel through connections. Constraints (3) assure that flows only go through the connections that are selected. Constraints (4) guarantee steady-state conditions at each connection. Constraints (5) are the non-negativity and the integrality constraints. We can see that the objective function is nonlinear in $\boldsymbol{x}$ and the problem formulation is a mixed integer nonlinear program (MINLP). 


\subsection{Convexity Property}

In this subsection, we establish that the objective function is convex in $\boldsymbol{x}, \boldsymbol{y}$. We can rewrite the objective function as:

$Z^{P_{1}}(\boldsymbol{x}, \boldsymbol{y})=\sum_{(i, j) \in A} \sum_{k \in K} \alpha f_{i j}\left(\frac{d_{i k}+d_{k j}}{v}\right) x_{i j k}+\alpha \sum_{k \in K} \frac{\bar{S}_{k}^{2}}{2} \frac{\left(\sum_{(i, j) \in A} f_{i j} x_{i j k}\right)^{2}}{1-\bar{S}_{k} \sum_{(i, j) \in A} f_{i j} x_{i j k}}+\sum_{k \in K}\left(F_{k} y_{k}+\alpha \bar{S}_{k} \sum_{(i, j) \in A} f_{i j} x_{i j k}\right)$

The convexity proof is based on a result in Bector (1968) that the ratio of a square of a nonnegative convex function and a positive concave function is convex.

Theorem 1: The function $Z^{P_{1}}(\boldsymbol{x}, \boldsymbol{y})$ is convex in $\boldsymbol{x}, \boldsymbol{y}$ if the inequalities stated in (4) hold.

Proof: If we can prove that

$$
\frac{\left(\sum_{(i, j) \in A} f_{i j} x_{i j k}\right)^{2}}{1-\bar{S}_{k} \sum_{(i, j) \in A} f_{i j} x_{i j k}}
$$

is convex in $\boldsymbol{x}$, then it is easy to see that $Z^{P_{1}}(\boldsymbol{x}, \boldsymbol{y})$ is convex in $\boldsymbol{x}, \boldsymbol{y}$. Let $f(\boldsymbol{x})=$ $\sum_{(i, j) \in A} f_{i j} x_{i j k}$ and $g(\boldsymbol{x})=1-\bar{S}_{k} \sum_{(i, j) \in A} f_{i j} x_{i j k}$. Then $f(\boldsymbol{x})$ is convex and $g(\boldsymbol{x})$ is concave with positive value because of constraints (4). We conclude from the result in Bector (1968) that

$$
\frac{\left(\sum_{(i, j) \in A} f_{i j} x_{i j k}\right)^{2}}{1-\bar{S}_{k} \sum_{(i, j) \in A} f_{i j} x_{i j k}}
$$

is convex. The theorem follows.

Now, we can see that the binary variables $\boldsymbol{y}$ are linear and separable from the continuous variables $\boldsymbol{x}$, the constraints are linear, and the objective function is convex for fixed values of the discrete variables. 


\subsection{Outer Approximation Algorithm}

As we mentioned before, the problem formulation $\left(P_{1}\right)$ is a mixed integer nonlinear optimization problem (MINLP). Major algorithms for solving the MINLP problem include: branch and bound (Beale (1977)), generalized Benders decomposition (GBD) (Geoffrion (1972)), and outer approximation (OA) (Duran and Grossman (1986)). Other approximating methods include: replacing the integer variables by continuous variables and approximating the nonlinear functions by linear functions. The combinatorial search techniques such as simulated annealing and genetic algorithms have also been used to solve MINLP problems. Watkins and McKinney (1998) believe that GBD and OA are better if binary variables $\boldsymbol{y}$ are linear and separable from the continuous variables $\boldsymbol{x}$, when both accuracy and computational time are considered.

In this paper, the OA algorithm will be used to solve Problem $\left(P_{1}\right)$. The method can be summarized as follows: It generates an upper bound and a lower bound at each iteration. The upper bound results from the primal problem, which corresponds to the problem with fixed integer variables. The lower bound results from the master problem. The master problem is derived using primal information. As the iterations proceed, it is shown that the sequence of updated upper-bounds is nonincreasing, the sequence of lower bounds is nondecreasing, and that the sequence converge in a finite number of iterations. See Duran and Grossman (1986) and Floudas (1995). The detail of the OA algorithm can be found in the Appendix. The method is implemented through the use of the general algebraic modeling system (GAMS). In Section 6.1, we provide some numerical results. 


\section{The Variable Service Rate Case}

In this section, capacity is also determined through both the mean and second moment (or variance) of service time at each connection. However, we assume that the mean and second moment of service time at each connection are decision variables here. Changing the mean of service time can be implemented by assigning workforce, machines (e.g., fork-lift trucks in a distribution center), etc. Controlling variances is far more complicated than changing the mean of service time. Often the variances encountered in a distribution center can be the results of many factors in the system. These factors include distribution center layout, use of warehouse management systems, automated storage and retrieval systems (AS/RS), workforce training, scheduling, or demand forecasting, among others. For certain applications, to control variance, these factors must be identified and controlled. See Melnyk, Denzler, and Fredendall (1992) for more details.

We assume that the connection installation cost function includes two components: the

fixed cost related to the location, $F_{k}$, and the cost related to the capacity, $c_{1} / \bar{S}_{k}+c_{2} / \bar{S}_{k}^{2}$, where $c_{1}$ and $c_{2}$ are constants. The fixed cost is related to connection location, for example, the cost of leasing land. The latter is related to connection capacity. The estimation of $c_{1}$ could be found by a regression analysis (for example, see Ashayeri, Gelders, and Wassenhove (1985)). While we are not aware of existing literature that discusses the estimation of $c_{2}$, we believe that statistical techniques such as multifactor analysis of variance (ANOVA) and multiple regression could be applied for this purpose. As noted earlier, it is generally more difficult to reduce the variance than it is to reduce the mean, and we therefore expect $c_{2}>c_{1}$.

The goal of this version of the problem is to locate some number of $M / G / 1$ queueing 
connections, allocate flows to them, and decide the service capacity at each selected connection.

\subsection{Formulation}

The selection of stochastic connections with variable service rate problem can be formulated as follows:

$$
\begin{aligned}
&\left(P_{2}\right) \min _{\boldsymbol{x}, \boldsymbol{y}, \overline{\boldsymbol{S}}, \overline{\boldsymbol{S}}^{\mathbf{2}} Z^{P_{2}}\left(\boldsymbol{x}, \boldsymbol{y}, \overline{\boldsymbol{S}}, \overline{\boldsymbol{S}}^{\mathbf{2}}\right)}=\sum_{(i, j) \in A} \sum_{k \in K} \alpha f_{i j}\left(\frac{d_{i k}+d_{k j}}{v}+\frac{\bar{S}_{k}^{2} \sum_{(i, j) \in A} f_{i j} x_{i j k}}{2\left(1-\bar{S}_{k} \sum_{(i, j) \in A} f_{i j} x_{i j k}\right)}\right. \\
&\left.+\bar{S}_{k}\right) x_{i j k}+\sum_{k \in K} y_{k}\left(F_{k}+c_{1} / \bar{S}_{k}+c_{2} / \bar{S}_{k}^{2}\right) \\
& \sum_{k \in K} x_{i j k}=1, \quad \forall(i, j) \in A \\
& \text { subject to } x_{i j k} \leq y_{k}, \quad \forall(i, j) \in A, \forall k \in K \\
& \bar{S}_{k} \sum_{(i, j) \in A} f_{i j} x_{i j k} \leq 1-\epsilon, \quad \forall k \in K \\
& x_{i j k} \geq 0, y_{k} \in\{0,1\}, \bar{S}_{k} \geq 0, \bar{S}_{k}^{2} \geq 0, \quad \forall(i, j) \in A, k \in K
\end{aligned}
$$

The objective function (6) minimizes the sum of travel cost, average service time cost at the connections and the fixed installation cost of the connections. Constraints (7)-(9) are identical to constraints $(2)-(4)$ in Problem $\left(P_{1}\right)$. However, notice that $(9)$ is not linear since $\bar{S}_{k}$ is a decision variable. Constraints (10) are the non-negativity and the integrality constraints.

Rewriting the objective function of Problem $\left(P_{2}\right)$, we obtain:

$$
\begin{gathered}
Z^{P_{2}}\left(\boldsymbol{x}, \boldsymbol{y}, \overline{\boldsymbol{S}}, \overline{\boldsymbol{S}}^{\mathbf{2}}\right)=\left(\sum_{(i, j) \in A} \sum_{k \in K} \alpha f_{i j} \frac{d_{i k}+d_{k j}}{v} x_{i j k}+\sum_{k \in K} F_{k} y_{k}\right) \\
+\sum_{k \in K}\left(\frac{\left.\alpha \bar{S}_{k}^{2}\left(\sum_{(i, j) \in A} f_{i j} x_{i j k}\right)\right)^{2}}{2\left(1-\bar{S}_{k} \sum_{(i, j) \in A} f_{i j} x_{i j k}\right)}+\alpha \bar{S}_{k} \sum_{(i, j) \in A} f_{i j} x_{i j k}+\left(c_{1} / \bar{S}_{k}+c_{2} / \bar{S}_{k}^{2}\right) y_{k}\right) .
\end{gathered}
$$

This problem formulation is also a mixed integer nonlinear programming (MINLP). What complicates this version of the problem is that the objective function may not be convex or concave and constraints (9) are not linear in this case. 


\subsection{Properties}

In this subsection we develop some key properties for the problem. These properties can enable development of efficient solution methods.

\subsubsection{The Equal Utilization Rate Property}

The first property we seek to establish is that the utilization rates at the connections are identical for an optimal solution to $\left(P_{2}\right)$. In order to do this, we take the following line of attack:

- Define a subproblem $\left(S P_{1}\right)$ of $\left(P_{2}\right)$;

- Prove that $\left(S P_{1}\right)$ has equal utilization rate property;

- Prove that $\left(P_{2}\right)$ has equal utilization rate property.

Subproblem $\left(S P_{1}\right) \quad$ Suppose that $T$ is the total number of the selected connections in the network. For simplicity, we assume that connections $1,2, \cdots, T$ are selected. Each connection is assumed to be an $M / G / 1$ queueing system. $\Lambda$ is the given total system flow, which is equal to $\sum_{(i, j) \in A} f_{i j}$. Let $\lambda_{k}$ be the arrival rate at connection $k$, then $\sum_{k=1}^{k=T} \lambda_{k}=\Lambda$. Let $\rho_{k}$ be the system utilization rate of connection $k$. Then $\rho_{k}=\lambda_{k} \bar{S}_{k}$. In order to prove the equal utilization rate property, we use $\rho_{k}$ and $\lambda_{k}$ instead of $\bar{S}_{k}$ and $\lambda_{k}$ to formulate the cost function. We now define the subproblem $\left(S P_{1}\right)$ of $\left(P_{2}\right)$ as follows:

$$
\min _{\boldsymbol{\rho}, \boldsymbol{\lambda}, \overline{\boldsymbol{S}}^{\mathbf{2}}} Z^{S P_{1}}\left(\boldsymbol{\rho}, \boldsymbol{\lambda}, \overline{\boldsymbol{S}}^{\mathbf{2}}\right)=\sum_{k=1}^{k=T}\left(\frac{\alpha \lambda_{k}^{2} \bar{S}_{k}^{2}}{2\left(1-\rho_{k}\right)}+\alpha \rho_{k}+\frac{c_{1} \lambda_{k}}{\rho_{k}}+\frac{c_{2}}{\bar{S}_{k}^{2}}\right)
$$




$$
\begin{array}{r}
\text { subject to } \sum_{k=1}^{k=T} \lambda_{k}=\Lambda, \\
1-\rho_{k} \geq \epsilon, \quad \forall k \in\{1, \cdots, T\}, \\
\rho_{k} \geq 0, \lambda_{k} \geq 0, \bar{S}_{k}^{2} \geq 0, \forall k \in\{1, \cdots, T\} .
\end{array}
$$

The equal utilization rate property for $\left(S P_{1}\right)$ The Lagrange function associated with Problem $\left(S P_{1}\right)$ takes the form:

$$
L\left(\boldsymbol{\rho}, \boldsymbol{\lambda}, \bar{S}^{2}, \mu, \boldsymbol{\eta}\right)=\sum_{k=1}^{k=T}\left(\frac{\alpha \lambda_{k}^{2} \bar{S}_{k}^{2}}{2\left(1-\rho_{k}\right)}+\alpha \rho_{k}+\frac{c_{1} \lambda_{k}}{\rho_{k}}+\frac{c_{2}}{\bar{S}_{k}^{2}}\right)-\mu\left(\sum_{k=1}^{k=T} \lambda_{k}-\Lambda\right)-\sum_{k=1}^{k=T} \eta_{k}\left(1-\epsilon-\rho_{k}\right),
$$

where $\eta_{k}>0, \forall k \in\{1, \cdots, T\}$, and $\mu$ is unrestricted in sign. The first order necessary conditions for optimality in this problem are as follows:

$$
\begin{array}{r}
\nabla_{\boldsymbol{\rho}} L\left(\boldsymbol{\rho}, \boldsymbol{\lambda}, \overline{\boldsymbol{S}^{2}}, \mu, \boldsymbol{\eta}\right)=0, \\
\nabla_{\boldsymbol{\lambda}} L\left(\boldsymbol{\rho}, \boldsymbol{\lambda}, \overline{\boldsymbol{S}^{2}}, \mu, \boldsymbol{\eta}\right)=0, \\
\nabla_{\overline{\boldsymbol{S}^{2}}} L\left(\boldsymbol{\rho}, \boldsymbol{\lambda}, \overline{\boldsymbol{S}^{2}}, \mu, \boldsymbol{\eta}\right)=0, \\
\eta_{k}\left(1-\epsilon-\rho_{k}\right)=0, \quad \forall k .
\end{array}
$$

From (17) and (18), we obtain:

$$
\frac{\alpha \lambda_{k} \bar{S}_{k}^{2}}{1-\rho_{k}}+\frac{c_{1}}{\rho_{k}}=\mu
$$

and

$$
\frac{\alpha \lambda_{k}^{2}}{2\left(1-\rho_{k}\right)}=\frac{c_{2}}{\left(\bar{S}_{k}^{2}\right)^{2}}
$$

Rewriting (21), we obtain,

$$
\frac{\alpha \lambda_{k}^{2} \bar{S}_{k}^{2}}{2\left(1-\rho_{k}\right)}=\frac{c_{2}}{\bar{S}_{k}^{2}}
$$

or, 


$$
\frac{2 c_{2}}{\bar{S}_{k}^{2}}=\lambda_{k}\left(\frac{2 c_{2} \alpha}{1-\rho_{k}}\right)^{1 / 2}
$$

Substituting (23) into the objective function $Z^{S P_{1}}\left(\boldsymbol{\rho}, \boldsymbol{\lambda}, \overline{\boldsymbol{S}}^{\mathbf{2}}\right)$, we have the following problem:

$$
\begin{aligned}
& \left(S P_{1}^{\prime}\right) \quad \min _{\boldsymbol{\rho}, \boldsymbol{\lambda}} Z^{S P_{1}^{\prime}}(\boldsymbol{\rho}, \boldsymbol{\lambda})=\sum_{k=1}^{k=T}\left(\alpha \rho_{k}+\frac{c_{1} \lambda_{k}}{\rho_{k}}+\lambda_{k}\left(\frac{2 c_{2} \alpha}{1-\rho_{k}}\right)^{1 / 2}\right) \\
& \text { subject to } \quad \sum_{k=1}^{k=T} \lambda_{k}=\Lambda \text {, } \\
& 1-\rho_{k} \geq \epsilon, \quad \forall k \in\{1, \cdots, T\}, \\
& \rho_{k} \geq 0, \lambda_{k} \geq 0, \quad \forall k \in\{1, \cdots, T\} \text {. }
\end{aligned}
$$

Similarly, the Lagrange function associated with Problem $\left(S P_{1}^{\prime}\right)$ takes the form,

$$
L\left(\boldsymbol{\rho}, \boldsymbol{\lambda}, \mu^{\prime}, \boldsymbol{\eta}^{\prime}\right)=\sum_{k=1}^{k=T}\left(\alpha \rho_{k}+\frac{c_{1} \lambda_{k}}{\rho_{k}}+\lambda_{k}\left(\frac{2 c_{2} \alpha}{1-\rho_{k}}\right)^{1 / 2}\right)-\mu^{\prime}\left(\sum_{k=1}^{k=T} \lambda_{k}-\Lambda\right)-\sum_{k=1}^{k=T} \eta_{k}^{\prime}\left(1-\epsilon-\rho_{k}\right),
$$

where $\eta_{k}^{\prime}>0, \forall k$, and $\mu^{\prime}$ is unrestricted in sign. Using one of the first order necessary conditions for optimality, $\nabla_{\boldsymbol{\lambda}} L\left(\boldsymbol{\rho}, \boldsymbol{\lambda}, \mu^{\prime}, \boldsymbol{\eta}^{\prime}\right)=0$, we obtain,

$$
\frac{c_{1}}{\rho_{k}}+\left(\frac{2 c_{2} \alpha}{1-\rho_{k}}\right)^{1 / 2}=\mu^{\prime}, \quad \forall k \in\{1, \cdots, T\}
$$

Since the constants $c_{1}, c_{2}, \alpha$ and $\mu^{\prime}$ in (28) are all independent of $k, \rho_{k}$ is also independent of $k$ according to the necessary condition. This implies that if $\boldsymbol{\rho}$ is an optimal solution for Problem $\left(S P_{1}^{\prime}\right)$, all $\rho_{k}$ should be equal, or all connections have the same system utilization rate. Let $\rho$ be the common utilization rate. From (24), we obtain,

$$
Z^{S P_{1}^{\prime}}(\rho, \boldsymbol{\lambda})=\sum_{k=1}^{k=T}\left(\alpha \rho+\frac{c_{1} \lambda_{k}}{\rho}+\lambda_{k}\left(\frac{2 c_{2} \alpha}{1-\rho}\right)^{1 / 2}\right)=\alpha T \rho+\frac{c_{1} \Lambda}{\rho}+\Lambda\left(\frac{2 c_{2} \alpha}{1-\rho}\right)^{1 / 2}
$$

For simplicity, let $B=\Lambda\left(2 c_{2} \alpha\right)^{1 / 2}$, then, 


$$
Z^{S P_{1}^{\prime}}(\rho, \boldsymbol{\lambda})=Z(\rho)=\alpha T \rho+\frac{c_{1} \Lambda}{\rho}+\frac{B}{(1-\rho)^{1 / 2}}
$$

From (30), the optimal objective value of Problem $\left(S P_{1}^{\prime}\right)$ can be decided only by $\rho$. How to find optimal $\rho$ will be discussed in Section 5.2.2. Notice that $\lambda_{k}$ does not appear in the objective function but $\Lambda\left(=\sum_{k=1}^{k=T} \lambda_{k}\right)$ does. Therefore, the optimal solution for Problem $\left(S P_{1}^{\prime}\right)$ is not unique. In fact, suppose that $\rho^{*}$ is optimal solution from $(30)$, then for any given $\lambda_{k}$ such that $\sum_{k=1}^{k=T} \lambda_{k}=\Lambda$, the solution $\left(\boldsymbol{\rho}^{*}, \boldsymbol{\lambda}\right)$ is optimal to Problem $\left(S P_{1}^{\prime}\right)$.

Thus, the optimal solution of Problem $\left(S P_{1}\right)$ can be found by solving Problem $\left(S P_{1}^{\prime}\right)$ to obtain a solution for $\boldsymbol{\rho}, \boldsymbol{\lambda}$ and (23) to obtain $\overline{\boldsymbol{S}^{2}}$.

The equal utilization rate property for Problem $\left(P_{2}\right) \quad$ Recalling that $Z^{P_{2}}\left(\boldsymbol{x}, \boldsymbol{y}, \overline{\boldsymbol{S}}, \overline{\boldsymbol{S}^{2}}\right)$ (see (11)) includes the following two types of costs:

$$
\sum_{(i, j) \in A} \sum_{k \in K} \alpha f_{i j} \frac{d_{i k}+d_{k j}}{v} x_{i j k}+\sum_{k \in K} F_{k} y_{k}
$$

and

$$
\sum_{k \in K}\left(\frac{\left.\alpha \bar{S}_{k}^{2}\left(\sum_{(i, j) \in A} f_{i j} x_{i j k}\right)\right)^{2}}{2\left(1-\bar{S}_{k} \sum_{(i, j) \in A} f_{i j} x_{i j k}\right)}+\alpha \bar{S}_{k} \sum_{(i, j) \in A} f_{i j} x_{i j k}+\left(c_{1} / \bar{S}_{k}+c_{2} / \bar{S}_{k}^{2}\right) y_{k}\right) .
$$

The first is a "fixed charge facility location" type of cost. Since $\lambda_{k}=\sum_{(i, j) \in A} f_{i j} x_{i j k}$, the second one is the objective function of Problem $\left(S P_{1}\right)$ when connection locations are fixed. In fact, by the discussion above, this cost can be determined without specifying exact connection locations as long as the total number of selected connections is determined, i.e., $\sum_{k \in K} y_{k}$.

Now we prove the following theorem.

Theorem 2: The utilization rates at the connections are identical for an optimal solution to Problem $\left(P_{2}\right)$. 
Proof: Suppose that $\left(\boldsymbol{x}^{*}, \boldsymbol{y}^{*}, \overline{\boldsymbol{S}}^{*}, \overline{\boldsymbol{S}}^{\mathbf{2}}\right)$ is optimal for Problem $\left(P_{2}\right)$ and $T^{*}=\sum_{k \in K} y_{k}^{*}$. We can find a feasible solution that is at least as good as this one in which the utilization rates at the connections are identical.

Consider Problem $\left(S P_{1}\right)$. For $T^{*}$, we can obtain an optimal solution of Problem $\left(S P_{1}\right)$ by the method discussed above. Let $\rho^{*}$ represent the optimal utilization rate and $V\left(T^{*}\right)$ represent the optimal objective value of Problem $\left(S P_{1}\right)$. Also, let $\lambda_{k}=\sum_{(i, j) \in A} f_{i j} x_{i j k}^{*}$, $\bar{S}_{k}{ }^{\prime}=\rho^{*} / \lambda_{k}$. and determine ${\overline{S_{k}}}^{\prime}$ from (23). Since $0<\rho^{*}<1$, it is easy to verify that the solution $\left(\boldsymbol{x}^{*}, \boldsymbol{y}^{*}, \overline{\boldsymbol{S}}^{\prime},{\overline{\boldsymbol{S}^{2}}}^{\prime}\right)$ is a feasible solution for Problem $\left(P_{2}\right)$.

Since $V\left(T^{*}\right)$ is the optimal objective value of Problem $\left(S P_{1}\right)$ for $T^{*}$, we obtain:

$$
\begin{gathered}
Z^{P_{2}}\left(\boldsymbol{x}^{*}, \boldsymbol{y}^{*}, \overline{\boldsymbol{S}}^{*}, \overline{\boldsymbol{S}}^{\mathbf{2}^{*}}\right)=\sum_{(i, j) \in A} \sum_{k \in K} \alpha f_{i j} \frac{d_{i k}+d_{k j}}{v} x_{i j k}^{*}+\sum_{k \in K} F_{k} y_{k}^{*} \\
+\sum_{k \in K}\left(\frac{\alpha \bar{S}_{k}^{2}\left(\sum_{(i, j) \in A} f_{i j} x_{i j k}^{*}\right)^{2}}{2\left(1-\bar{S}_{k}^{*} \sum_{(i, j) \in A} f_{i j} x_{i j k}^{*}\right)}+\alpha \sum_{(i, j) \in A} f_{i j} x_{i j k}^{*} \bar{S}_{k}^{*}+y_{k}^{*}\left(c_{1} / \bar{S}_{k}^{*}+c_{2} / \bar{S}_{k}^{2^{*}}\right)\right) \\
\geq \sum_{(i, j) \in A} \sum_{k \in K} \alpha f_{i j} \frac{d_{i k}+d_{k j}}{v} x_{i j k}^{*}+\sum_{k \in K} F_{k} y_{k}^{*}+V\left(T^{*}\right) \\
=Z^{P_{2}}\left(\boldsymbol{x}^{*}, \boldsymbol{y}^{*}, \overline{\boldsymbol{S}}^{\prime}, \overline{\boldsymbol{S}}^{\mathbf{2}}\right) .
\end{gathered}
$$

The result follows.

Therefore, Problem $\left(P_{2}\right)$ can be solved as follows:

- Solve the following problem (see Section 5.3):

$$
\begin{gathered}
\left(P_{2}^{\prime}\right) \quad \min _{\boldsymbol{x}, \boldsymbol{y}} Z^{P_{2}^{\prime}}(\boldsymbol{x}, \boldsymbol{y})=\sum_{(i, j) \in A} \sum_{k \in K} \alpha f_{i j} \frac{d_{i k}+d_{k j}}{v} x_{i j k}+\sum_{k \in K} F_{k} y_{k}+V\left(\sum_{k \in K} y_{k}\right) \\
\text { subject to } \quad \sum_{k \in K} x_{i j k}=1, \quad \forall(i, j) \in A, \\
x_{i j k} \leq y_{k}, \quad \forall(i, j) \in A, \forall k \in K, \\
x_{i j k} \geq 0, y_{k} \in\{0,1\}, \quad \forall(i, j) \in A, k \in K .
\end{gathered}
$$


- Let $T=\sum_{k \in K} y_{k}$, find the common utilization rate $\rho$ (see Section 5.2.2).

- Let $\bar{S}_{k}=\frac{\rho}{\sum_{(i, j) \in A} f_{i j} x_{i j k}}$ and find $\bar{S}_{k}^{2}$ by $(23)$.

We now discuss the practical implications of the equal utilization property. Since the utilization rates at the connections must be identical, smaller values of total flow through

connection $k, \lambda_{k}$, would imply larger values of $\bar{S}_{k}$. This in turn could signify the degree of automation in information and material flow at the DC. More specifically, if a DC is dealing with low volume, it could have less automation which would lead to a higher mean service time. On the other hand, DCs dealing with high flow rates could reduce residence time by improved supply chain coordination.

\subsubsection{Finding the Common Utilization Rate}

Given the fact the utilization rate is identical for an optimal solution we now proceed to determine this optimal value. Consider the first and the second derivative of the objective function $Z(\rho)$ defined in (30):

$$
Z^{\prime}(\rho)=\alpha T-\frac{c_{1} \Lambda}{\rho^{2}}+\frac{B}{2(1-\rho)^{3 / 2}}
$$

and

$$
Z^{\prime \prime}(\rho)=\frac{2 c_{1} \Lambda}{\rho^{3}}+\frac{3 B}{4(1-\rho)^{5 / 2}} .
$$

We note that $Z^{\prime \prime}>0$ for $\rho \in(0,1)$ since $B, c_{1}$, and $\Lambda$ are positive, implying that the function $Z$ is convex. Thus the candidate optima for $\rho$ are the roots of $g(\rho)=Z^{\prime}(\rho)=0$. An iterative technique such as bisection method can be used for this purpose. 
The properties of $g(\rho)$ are given by the following theorem.

Theorem 3: The objective function $g(\rho)$ has the following properties within the interval $(0,1)$ :

(a) $g(\rho)$ is a strictly increasing function;

(b) equation $g(\rho)=0$ has a unique root;

(c) when the total number of connections, $T$, increases, the optimal value of $\rho$ will decrease.

Proof: Since $Z^{\prime \prime}>0$ as discussed earlier, (a) is proved. Now, from $g(0)=-\infty, g(1)=\infty$ and (a), we know that equation $g(\rho)=0$ has a unique root within the interval $(0,1)$. (b) is proved.

In order to prove (c), consider the equation $g(\rho)=0$ and (for convenience) treat $T$ as a continuous variable. From the derivative with respect to $T$ we obtain,

$$
\frac{d \rho}{d T}=-\frac{\alpha}{3 B / 4(1-\rho)^{5 / 2}+2 c_{1} \Lambda / \rho^{3}}<0 .
$$

Thus (c) is true for continuous $T$. So, (c) is also true if $T$ is discrete.

From (29) and the above theorem, we can see that when the total number of selected connections increases, $Z^{S P_{1}}\left(\rho, \boldsymbol{\lambda}, \overline{\boldsymbol{S}}^{\mathbf{2}}\right)$ will increase, but the optimal system utilization rate $\rho$ will become smaller.

\subsubsection{Upper Bounds for Total Number of Connections and Total Service Rate}

In this subsection, we try to find upper bounds for the total number of connections and total service rate in the system. Consider the following uncapacitated fixed charge connection location problem: 


$$
\begin{gathered}
\left(S P_{2}\right) \min _{\boldsymbol{x}, \boldsymbol{y}} Z^{S P_{2}}(\boldsymbol{x}, \boldsymbol{y})=\sum_{(i, j) \in A} \sum_{k \in K} \alpha f_{i j} \frac{d_{i k}+d_{k j}}{v} x_{i j k}+\sum_{k \in K} F_{k} y_{k} \\
\text { subject to } \sum_{k \in K} x_{i j k}=1, \quad \forall(i, j) \in A, \\
x_{i j k} \leq y_{k}, \quad \forall(i, j) \in A, \forall k \in K, \\
x_{i j k} \geq 0, y_{k} \in\{0,1\}, \quad \forall(i, j) \in A, k \in K .
\end{gathered}
$$

Let $\left(\boldsymbol{x}^{*}, \boldsymbol{y}^{*}\right)$ be the optimal solution for Problem $\left(S P_{2}\right)$ and $T^{*}=\sum_{k \in K} y_{k}^{*}$, then, we have the following theorem.

Theorem 4: The upper bound for total number of connections in Problem $\left(P_{2}\right)$ is $T^{*}$.

Proof: Consider Problem $\left(P_{2}^{\prime}\right)$. Since $\left(\boldsymbol{x}^{*}, \boldsymbol{y}^{*}\right)$ is the optimal solution for Problem $\left(S P_{2}\right)$, opening one more connections will not decrease the objective value of Problem $\left(S P_{2}\right)$. On the other hand, from (30), we know that $V(T)$ is an increasing function of $T$. Verifying (31), the result follows.

Let $\rho^{*}$ be the optimal utilization rate for this $T^{*}$. From Theorem 4 (c), we have the following corollary:

Corollary 1: $\rho^{*}$ is the lower bound for the optimal utilization rate of Problem $\left(P_{2}\right)$.

Since $V\left(\sum_{k \in K} y_{k}\right)$ in Problem $\left(P_{2}^{\prime}\right)$ is not affected by $\boldsymbol{\lambda}, \overline{\boldsymbol{S}}^{\mathbf{2}}$, we can always find the average service time $\bar{S}_{k}$ or the average service rate $1 / \bar{S}_{k}$ by $\bar{S}_{k}=\rho / \sum_{(i, j) \in A} f_{i j} x_{i j k}$. Thus,

$$
\sum_{k \in K} \frac{1}{\bar{S}_{k}}=\sum_{k \in K} \frac{\sum_{(i, j) \in A} f_{i j} x_{i j k}}{\rho}=\frac{\sum_{k \in K} \sum_{(i, j) \in A} f_{i j} x_{i j k}}{\rho}=\frac{\Lambda}{\rho}
$$

where $\Lambda=\sum_{(i, j) \in A} f_{i j}$ is the given total system flows. Thus we obtain:

Corollary 2: The upper bound for the optimal total service rate of Problem $\left(P_{2}\right)$ is $\Lambda / \rho^{*}$. 
Corollary 3: If $\rho^{*}$ is the optimal utilization rate of Problem $\left(P_{2}\right), \Lambda / \rho^{*}$ is the optimal total service rate of Problem $\left(P_{2}\right)$.

\subsection{Lagrangian Relaxation Algorithm}

In this section, based on some of the previous properties, we develop a Lagrangian relaxation algorithm to solve Problem $\left(P_{2}^{\prime}\right)$. Let $(L R P)$ be the Lagrangian relaxation problem corresponding to relaxing the assignment constraints (32) for a given set of Lagrangian multipliers

$\mu$.

$$
(L R P) \min _{\boldsymbol{x}, \boldsymbol{y}} Z^{L R P}(\boldsymbol{\mu})=\sum_{(i, j) \in A} \sum_{k \in K} \alpha f_{i j} \frac{d_{i k}+d_{k j}}{v} x_{i j k}+\sum_{k \in K} y_{k} F_{k}+V\left(\sum_{k \in K} y_{k}\right)+\sum_{(i, j) \in A} \mu_{i j}\left(1-\sum_{k \in K} x_{i j k}\right)
$$

subject to (33) and (34).

The objective function of $(L R P)(\boldsymbol{\mu})$ can be rewritten as:

$$
(L R P) \min _{\boldsymbol{x}, \boldsymbol{y}} Z^{L R P}(\boldsymbol{\mu})=\sum_{k \in K} \sum_{(i, j) \in A}\left(\alpha f_{i j} \frac{d_{i k}+d_{k j}}{v}-\mu_{i j}\right) x_{i j k}+\sum_{k \in K} y_{k} F_{k}+V\left(\sum_{k \in K} y_{k}\right)+\sum_{(i, j) \in A} \mu_{i j}
$$

The ideal choice of multipliers is such that they solve the Lagrangian dual problem, denoted by $(D P)$ :

$$
(D P) \quad \max _{\boldsymbol{\mu}} Z^{D P}(\boldsymbol{\mu})
$$

The optimal value of the above problem $(D P)$ provides the "best" lower bound (using the Lagrangian method).

For fixed values of the Lagrange multipliers $\boldsymbol{\mu}$, the optimal solution for $(L R P)$ can be obtained by the following method:

Calculate $p_{k}=F_{k}+\sum_{(i, j) \in A} \min \left\{0,\left(\alpha f_{i j} \frac{d_{i k}+d_{k j}}{v}-\mu_{i j}\right)\right\}$ for each $k$. Sort $p_{k}$ in increasing 
order and let $p_{k_{1}}, p_{k_{2}}, \cdots$ be the sequence after sorting, so that $p_{k_{1}} \leq p_{k_{2}} \leq \cdots$. Let

$$
\Delta_{V(T)}= \begin{cases}V(0), & \text { if } T=0 \\ V(T)-V(T-1), & \text { if } T>0\end{cases}
$$

From the Section 5.2.2, $\Delta_{V(T)} \geq 0$. The optimal $\boldsymbol{y}$ value can be obtained by the following procedure:

Set $y_{k_{1}}=1$;

For $j=2$ to $|K|$

set $y_{k_{j}}=1$, if $p_{k_{j}}+\Delta_{V}(j)<0$;

set $y_{k_{j}}=0$, otherwise.

The corresponding optimal $\boldsymbol{x}$ value is

$$
x_{i j k}= \begin{cases}1, & \text { if } y_{k}=1 \text { and } \alpha f_{i j} \frac{d_{i k}+d_{k j}}{v}-\mu_{i j}<0 \\ 0, & \text { otherwise. }\end{cases}
$$

If the solution for the relaxed problem is feasible to the original problem, we compute and update the upper bound of the original problem. If it is not, i.e., some flows are assigned to more than one connection or some flows are not assigned to any connection, we can construct a primal feasible solution by simply opening connections with $y_{k}=1$ and assigning flows to their closest connections. The primal objective will provide us with an upper bound on the solution. If the termination conditions are satisfied, we stop. Otherwise, we decide if we need to update the Lagrange multipliers $\mu_{i j}$. If we decide to terminate the Lagrangian procedure, the best upper bound gives us a heuristic solution. The following is a step-by-step description of the procedure:

Step 0. Prepare $V(T)$ and $\Delta_{V}(T)$ for $T \in K$. 
Step 1. Relax constraints (32), fix the initial $\mu_{i j}$ (we arbitrarily choose $\mu_{i j}=1$ here);

Step 2. Solve $(L R P)$, compute and update the lower bound;

Step 3. If the solution for the relaxed problem is feasible to $\left(P_{2}^{\prime}\right)$, update the upper bound; if not, find a feasible solution by the method mentioned above, then update the upper bound;

Step 4. If the termination conditions are satisfied, stop; otherwise, update $\mu_{i j}$ (see Fisher (1981)), and go to Step 2.

\section{Computational Performance}

In this section, we present the results of randomly generated problems for both cases. We first generated each node's location, which was decided by its $x$-coordinate and the $y$-coordinate. These coordinate values were randomly selected from $U(0,1000)$, where $U$ denotes a uniform distribution. The candidate sites of connections were also decided by its $x$-coordinate and the $y$-coordinate, where the $x$-coordinate values and $y$-coordinate values were randomly selected from the minimum value of the $x$-coordinate and the $y$-coordinate values of node's location to maximum value of $x$-coordinate and the $y$-coordinate values of node's location. For each pair of distinct nodes, the amount of flow was randomly drawn from $U(5,30) . F_{k}$ is randomly selected from $U(3000,5000)$. We assume $\alpha=1$ and $v=1$ in both cases. To demonstrate the performance of the algorithms, we use both "heuristic gap" and CPU time to evaluate the efficiency of our algorithm. Heuristic gap is defined as (best upper bound - best lower bound)/best lower bound * 100. Using this term, we can guarantee that the solution for the 
original problem is near-optimal if the heuristic gap is very small.

\subsection{OA Algorithm}

In the fixed service rate case, we need to generate the mean service time $\bar{S}_{k}$ and the second moment of service time $\bar{S}_{k}^{2}$. Let $\psi=\sum_{(i, j) \in A} f_{i j} /|K|$, where $|K|$ is the total number of candidate connection sites and $\psi$ is the average flow for the total number of candidate connection sites. The service rate $1 / \bar{S}_{k}$ is randomly selected from $U(3 \psi, 4 \psi)$. We let $\bar{S}_{k}^{2}=$ $2 \bar{S}_{k}$. The OA algorithm was implemented through the use of GAMS using its default settings. The MILP solver used was CPLEX, and the NLP solver was CONOPT2. The procedure stops as soon as the bound defined by the objective of the last MILP master problem is worse than the best NLP solution found (a "crossover" occurred) or the number of cycles is greater than 50 .

Table 1 shows the problem size, CPU times for NLP and MILP, and heuristic gap. Only relatively small size problem can be solved by GAMS. Also, we note that the heuristic gap increases as the problem size increases.

Table 1: Computational result for OA algorithm

\begin{tabular}{|l|c|c|r|r|r|c|}
\hline & Flow \# & Connection \# & Node \# & \multicolumn{2}{|c|}{ CPU $(\mathrm{s})$} & \multirow{2}{*}{ Heuristic gap (\%) } \\
\cline { 5 - 6 } & & & & NLP & MILP & \\
\hline 1 & 45 & 15 & 10 & 1.91 & 1.91 & 0.74 \\
2 & 105 & 20 & 15 & 199.45 & 195.76 & 1.23 \\
3 & 190 & 30 & 20 & 2289.37 & 1282.48 & 1.45 \\
4 & 300 & 40 & 25 & 10878.62 & 3229.64 & 2.50 \\
\hline
\end{tabular}




\subsection{Lagrangian Relaxation Algorithm}

In the variable service rate case, we arbitrarily choose $c_{1}=1$ and $c_{2}=1$. The bisection method is used to find $V(T)$ for each $T$. We terminate the procedure if the heuristic gap is less than 0.0001 or after 1000 iterations. The algorithm is coded in $\mathrm{C}++$.

Table 2 shows that the algorithm performs extremely well for all the test problems. From the table, we can see the average heuristic gap for all test problems is about $0.22 \%$ and the CPU times are less than 8 minutes for all size problems.

Table 2: Computational result for LR algorithm

\begin{tabular}{|l|c|c|r|r|c|}
\hline & Flow \# & Connection \# & Node \# & CPU (s) & Heuristic gap (\%) \\
\hline 1 & 45 & 15 & 10 & 0.05 & 0.01 \\
2 & 190 & 30 & 20 & 3.56 & 0.13 \\
3 & 435 & 45 & 30 & 12.37 & 0.02 \\
4 & 1485 & 110 & 55 & 113.35 & 0.22 \\
5 & 1770 & 130 & 60 & 165.58 & 0.38 \\
6 & 2080 & 160 & 65 & 260.50 & 0.47 \\
7 & 2415 & 180 & 70 & 324.32 & 0.16 \\
8 & 2775 & 190 & 75 & 404.58 & 0.44 \\
9 & 3160 & 200 & 80 & 461.87 & 0.19 \\
\hline
\end{tabular}

\section{Summary and Future Work}

This paper addresses the problem of selecting and sizing $M / G / 1$ queueing connections in a distribution network. Mathematical programming formulations are presented for both the fixed and variable service rate cases. For the fixed service rate case, we prove that the objective function is convex and use this result to present an outer approximation (OA) 
algorithm. The algorithm was implemented through the use of GAMS. For the variable service rate case, we establish that the utilization rates at the connections are identical for an optimal solution. Based on this key property, we develop a Lagrangian relaxation algorithm. Compared to the OA algorithm, this algorithm performs very well under both the accuracy and CPU time criteria. A useful extension to the work presented in this paper would be to modify the models and solution approaches to treat capacity as a set of discrete options. Modeling the connections as $M / G / k$ queueing systems could be another challenging extension.

\section{Appendix}

The OA algorithm as applied to the problem $\left(P_{1}\right)$ can be stated as follows:

Step 1 : Select initial values for variables $\boldsymbol{y}$, say, $\boldsymbol{y}^{1}=\mathbf{1}$. Set the counter $m=1$ and the current upper bound $U B D=+\infty$. Choose the convergence tolerance $\delta$.

Step 2 : Solve the primal problem PP:

$$
\begin{aligned}
& (P P) \min _{\boldsymbol{x}} Z^{P P}(\boldsymbol{x})=\sum_{(i, j) \in A} \sum_{k \in K} \alpha f_{i j}\left(\frac{d_{i k}+d_{k j}}{v}+\frac{\bar{S}_{k}^{2} \sum_{(i, j) \in A} f_{i j} x_{i j k}}{2\left(1-\bar{S}_{k} \sum_{(i, j) \in A} f_{i j} x_{i j k}\right)}+\bar{S}_{k}\right) x_{i j k}+\sum_{k \in K} F_{k} y_{k}^{m} \\
& \text { subject to } \quad \sum_{k \in K} x_{i j k}=1, \quad \forall(i, j) \in A \text {, } \\
& x_{i j k} \leq y_{k}^{m}, \quad \forall(i, j) \in A, \forall k \in K, \\
& 1-\bar{S}_{k} \sum_{(i, j) \in A} f_{i j} x_{i j k} \geq \epsilon, \quad \forall k \in K, \\
& x_{i j k} \geq 0, \quad \forall(i, j) \in A, k \in K \text {. }
\end{aligned}
$$

It gives an optimal primal solution $\boldsymbol{x}^{m}$ and an optimal value of objective function $Z\left(\boldsymbol{x}^{m}, \boldsymbol{y}^{m}\right)$. Update the current upper bound $U B D=\min \left\{U B D, Z\left(\boldsymbol{x}^{m}, \boldsymbol{y}^{m}\right)\right\}$. If 
$U B D=Z\left(\boldsymbol{x}^{m}, \boldsymbol{y}^{m}\right)$, set $\boldsymbol{y}^{*}=\boldsymbol{y}^{m}$ and $\boldsymbol{x}^{*}=\boldsymbol{x}^{m}$, where $\boldsymbol{x}^{*}, \boldsymbol{y}^{*}$ is the current best solution.

Step 3 : Solve the following master problem:

$$
(M P) \min _{\boldsymbol{y}, \eta} Z^{M P}(\boldsymbol{y}, \eta)=\eta+\sum_{k \in K} F_{k} y_{k}
$$

subject to

$$
\begin{array}{r}
\eta \geq f\left(\boldsymbol{x}^{m}\right)+\nabla f\left(\boldsymbol{x}^{m}\right)^{T}\left(\boldsymbol{x}-\boldsymbol{x}^{m}\right), \forall m \in\{1,2, \cdots, M\}, \\
\sum_{k \in K} x_{i j k}=1, \quad \forall(i, j) \in A, \\
x_{i j k} \leq y_{k}, \quad \forall(i, j) \in A, \forall k \in K, \\
1-\bar{S}_{k} \sum_{(i, j) \in A} f_{i j} x_{i j k} \geq \epsilon, \quad \forall k \in K, \\
\eta \in R^{1}, x_{i j k} \geq 0, y_{k} \in\{0,1\}, \quad \forall(i, j) \in A, k \in K .
\end{array}
$$

Where $f(\boldsymbol{x})=\sum_{(i, j) \in A} \sum_{k \in K} \alpha f_{i j}\left(\frac{d_{i k}+d_{k j}}{v}+\frac{\bar{S}_{k}^{2} \sum_{(i, j) \in A} f_{i j} x_{i j k}}{2\left(1-\bar{S}_{k} \sum_{(i, j) \in A} f_{i j} x_{i j k}\right)}+\bar{S}_{k}\right) x_{i j k}$. The solution of the master problem gives $\boldsymbol{y}^{m+1}$ and $Z^{M P}$, where $Z^{M P}$ is a lower bound on the objective function of $\left(P_{1}\right)$. If $\left(U B D-Z^{M P}\right) / U B D \leq \epsilon$, stop, the solution is $\boldsymbol{x}^{*}, \boldsymbol{y}^{*}$; otherwise, set $m=m+1$ and return to step 2 .

\section{Acknowledgments}

The authors would like to acknowledge support from the National Science Foundation via Grant No. DMI-0300370. 


\section{References}

Ashayeri, J., L. F. Gelders, and L. V. Wassenhove (1985). A microcomputer-based optimization model for the design of automated warehouses. International Journal of Production Research 23, 825-839.

Aykin, T. (1994). Lagrangian relaxation based approaches to capacitated hub-and-spoke network design problem. European Journal of Operational Research 79, 501-523.

Batta, R., A. Ghose, and U. S. Palekar (1989). Locating facilities on the Manhattan metric with arbitrary shaped barriers and convex forbidden regions. Transportation Science 23, $26-36$.

Beale, E. M. L. (1977). Integer programming. In D. Jacobs (Ed.), The State of the Art in Numerical Analysis, pp. 409. Academic Press.

Bector, C. R. (1968). Programming problems with convex fractional functions. Operations Research 16, 383-391.

Berman, O. and R. C. Larson (1982). The median problem with congestion. Computers and Operations Research 9, 119-126.

Berman, O., R. C. Larson, and N. Fouska (1992). Optimal location of discretionary service facilities. Transportation Science 26, 201-211.

Berman, O., R. C. Larson, and C. Parkan (1987). The stochastic queue p-median problem. Transportation Science 21, 207-216.

Bitran, G. B. and D. Tirupati (1989). Tradeoff curves, targeting and balancing in manufacturing queueing networks. Operations Research 37, 547-564. 
Bretthauer, K. M. (1995). Capacity planning in networks of queues with manufacturing applications. Mathl. Comput. Modelling 21, 35-46.

Brimberg, J. and A. Mehrez (1997). A note on the allocation of queueing facilities using a minisum criterion. Journal of the Operational Research Society 48, 195-201.

Brimberg, J., A. Mehrez, and G. O. Wesolowsky (1997). Allocation of queueing facilities using a minimax criterion. Location Science 5, 89-101.

Campbell, J. F. (1994). Integer programming formulations of discrete hub location problems. European Journal of Operational Research 72, 387-405.

Crabill, T. B., D. Gross, and M. J. Magazine (1977). A classified bibliography of research on optimal design and control of queues. Operations Research 25, 219-232.

Duran, M. A. and I. E. Grossman (1986). An outer approximation algorithm for a class of mixed-integer nonlinear programs. Mathematical Programming 36, 307-339.

Ebery, J., M. Krishnamoorthy, A. Ernst, and N. Boland (2000). The capacitated multiple allocation hub location problem: Formulations and algorithms. European Journal of Operational Research 120, 614-631.

Enns, S. T. (1998). Work flow analysis using queuing decomposition models. Computers and Industrial Engineering 34, 371-383.

Fisher, M. L. (1981). The lagrangian relaxation method for solving integer programming problem. Management Science 27, 1-18.

Floudas, C. A. (1995). Nonlinear and Mixed-Integer Optimization: Fundamentals and Applications. Oxford University Press, New York. 
Geoffrion, A. M. (1972). Generalized benders decomposition. J. Opt. Theory and Appl. 10, $237-260$.

Gross, D. and C. M. Harris (1998). Fundamentals of Queueing Theory, 3rd Edition. John Wiley, New York.

Hillier, F. S. and K. C. So (1991). The effect of the coefficient of variation of operation times on the allocation of storage space in production line systems. IIE Transactions 23, $198-206$.

Hodgon, M. J. (1990). A flow-capturing location-allocation model. Geographical Analysis 22, 270-279.

Huang, S., R. Batta, and R. Nagi (2003). Variable capacity sizing and selection of connections in a facility layout. IIE Transactions 35, 49-59.

Marianov, V. (2003). Location of multiple-server congestible facilities for maximizing expected demand when services are non-essential. Annals of Operations Research 123, 125141.

Melnyk, S. A., D. R. Denzler, and L. Fredendall (1992). Variance control vs. dispatching efficiency. Production and Inventory Management Journal Third Quarter, 6-13.

O'Kelly, M. (1986). The location of interacting hub facilities. Transportation Science 20, $92-106$.

Park, C. S. (2002). Contemporary Engineering Economics, 3rd Edition. Prentice-Hall, NJ. Shanthikumar, J. D. and D. D. Yao (1988). On server allocation in multiple center manufacturing systems. Operations Research 36, 333-342. 
Stidham, S. (2002). Analysis, design, and control of queueing systems. Operations Research 50, 197-216.

Wang, Q., R. Batta, and C. M. Rump (2002). Algorithms for a facility location problem with stochastic customer demand and immobile servers. Annals of Operations Research 111, 17-34.

Watkins, D. W. and D. C. McKinney (1998). Decomposition methods for water resources optimization models with fixed costs. Advances in Water Resources 21, 283-295.

Yamazaki, G., H. Sakasegawa, and J. G. Shanthikumar (1992). On the optimal arrangement of stations in tandem queueing system with blocking. Management Science 39, $137-153$.

Yao, D. D. and S. C. Kim (1987). Reducing the congestion in a class of job shops. Management Science 34, 1165-1172. 